\title{
The Pericardial Body of Ciona intestinalis Contains Hemocytes and Degenerating Muscle Cells, But No Parasites
}

\author{
Lilly Rohlfs ${ }^{1,2} \cdot$ Katja Müller $^{1} \cdot$ Thomas Stach $^{2}$ (])
}

Received: 11 June 2020 / Accepted: 28 November 2020 / Published online: 26 December 2020

(c) The Author(s) 2020

\begin{abstract}
Purpose A ventral heart positioned posterior to the branchial basket and equipped with a pericardium is homologous in tunicates and their sister group, the craniates, yet the tunicate model organism Ciona intestinalis features a pericardial body, a structure peculiar to few ascidian species. Here, we set out to distinguish between two competing hypotheses regarding the function of the pericardial body found in the literature: $\left(\mathrm{H}_{1}\right)$ The pericardial body performs a role in the removal of dysfunctional myocardial cells, and $\left(\mathrm{H}_{2}\right)$ it is a specialized niche of the immune system involved in defense against parasites. Methods We used histological techniques, transmission electron microscopy, and PCR-based gene sequencing to investigate whether individual ascidians parasitized with apicomplexan protists show signs of infections within the pericardial body.

Results In individuals of $C$. intestinalis from the German North Sea infested with apicomplexan protists, the pericardial body contains numerous myocardial cells in various stages of degeneration while no remnants of parasitic cells could be identified. Conclusion Thus, we conclude that $\mathrm{H}_{2}$ - the pericardial body is a specialized niche of the immune system involved in defense against parasites - can be refuted. Rather, our observations support $\mathrm{H}_{1}$, the hypothesis that the pericardial body performs a role in the removal of dysfunctional myocardial cells.
\end{abstract}

Keywords Gregarines · Acidian · Tunicate $\cdot$ Marine invertebrate

\section{Introduction}

Generations of German-speaking morphologists have been introduced to the diversity of animal body plans with Kükenthal's "Leitfaden für das zoologische Praktikum" as the principal textbook. The first edition came into print in 1898 [16] and its current 27th edition (under the name "Kükenthal-Zoologisches Praktikum") with Volker Storch and Ullrich Welsch as authors is still the basis of many zoology courses at the university level [34]. One of the subjects of study in this book is the sea squirt Ciona intestinalis, now

Supplementary Information The online version contains supplementary material available at https://doi.org/10.1007/s1168 6-020-00323-x.

Thomas Stach

thomas.stach@hu-berlin.de

1 Humboldt-Universität zu Berlin, Institut für Biologie, Molekulare Parasitologie, Berlin, Germany

2 Humboldt-Universität zu Berlin, Institut für Biologie, Vergleichende Elektronenmikroskopie, Berlin, Germany a well-known model organism for molecular developmental studies [20]. Starting with the 20th edition until today, in the chapter on $C$. intestinalis, aspiring morphologists stumble upon the didactically engaging and beautifully phrased sentence 'Im Herz findet sich regelmäßig ein weißes "Bällchen" unbekannter Funktion' (within the heart a little white ball of unknown function is regularly found-translation TS). Students, who too often assume that everything is known in morphology, are dependably inspired by this challenge. However, despite this prominent placement in an eminent textbook-what is the actual state of knowledge concerning this 'little white ball' in the primary literature?

In the zoological primary literature, the 'little white ball' is known as pericardial (or sometimes pericardic) body. The statement cited above, which locates the pericardial body in the heart, is not precise because the pericardial body is in fact situated within the pericardium. However, the main point, i.e., that the function remains unknown, is fundamentally correct, and primarily two competing hypotheses for its function are suggested. According to hypothesis $1\left(\mathrm{H}_{1}\right)$, the pericardial body is the site of regulated degradation of senescent myocardial cells. The alternative hypothesis $\left(\mathrm{H}_{2}\right)$ 
suggests that the pericardial body plays a crucial role in parasite removal as a central part of the immune system. $\mathrm{H}_{1}$ is based on the early light microscopical observation by Roule [27] that the pericardial body contained myocytes, and this observation was further supported in a study by Fernandez [12] and Millar [23]. However, because Fernandez astutely observed different types of hemocytes and erroneously thought the myocardial cells needed to transgress the epithelium of the pericardium, he nevertheless cast doubt on Roule's original observations. Although ultrastructural observations confirmed the presence of degenerating myocytes and hemocytes in the pericardial body (e.g., Scippa and Izzo [31]), this research group later also observed apicomplexan, intracellular parasites in hemocytes mainly in the periphery of the pericardium of $C$. intestinalis species from the gulf of Naples [6, 32]. This observation led the authors suggest $\mathrm{H}_{2}$, i.e., that the pericardial body is a site of passive collection of these parasites. According to this hypothesis, the presence of myocardial cells in the pericardial body is a secondary result of their physical disruption caused by the constant passive movement of the pericardial body against the pericardial wall.

Of course, the two hypotheses are not mutually exclusive and the pericardial body might in fact serve a combination of both functions. In any case, while both hypotheses are in agreement with some observations, no study so far has shown the infestation with parasites simultaneously to an investigation of the ultrastructural morphology of the pericardial body. We argue; however, that this strategy would strengthen one of the hypotheses because.

If the myocyte-removal-hypothesis $\left(\mathrm{H}_{1}\right)$ is true, then even in infected specimens, there should be ultrastructural evidence of degenerating muscle cells only in the pericardial body.

If, on the other hand, the parasitism hypothesis $\left(\mathrm{H}_{2}\right)$ is true, then in infected specimens, there should be ultrastructural evidence of parasites in the pericardial body.

Thus, in order to distinguish between the two hypotheses, we studied several specimens of Ciona intestinalis for the presence of parasites using microscopic and molecular identification. We subsequently investigated the pericardial bodies of $C$. intestinalis specimens positively infected with gregarines using histology and transmission electron microscopy.

\section{Materials and Methods}

\section{Animal Maintenance and Anesthetization}

Living specimens of Ciona intestinalis (Linnaeus, 1767) were supplied in July 2018 by mail from the Biological Marine Station at the Alfred-Wegener-Institute on
Helgoland. Animal size ranged from 5 to $12 \mathrm{~cm}$ when relaxed, i.e., animals were mature yet not fully grown. On Helgoland, only $C$. intestinalis is known to occur [33] and characteristics of the specimens used in the current study confirmed the species identity [3]. Animals were kept for up to 4 weeks in seawater tanks at $16-18^{\circ} \mathrm{C}$, ambient light and fed with approximately $1 \mathrm{ml}$ Liquifry marine (Interpet Ltd., UK) per 101 of seawater. Before further preparation, animals were anesthetized, by placing them individually in a 11 glass beaker filled with micro-filtered $(0.4-\mu \mathrm{m}$ pore size $)$ seawater. $2-3$ crystals of menthol were added and the beaker containing animals, seawater, and menthol was placed in a refrigerator at $4{ }^{\circ} \mathrm{C}$ for $30 \mathrm{~min}$.

\section{Electron Microscopy}

Freshly dissected pericardial bodies were prefixed in $2.5 \%$ glutaraldehyde, $2 \%$ paraformaldehyde in $0.2 \mathrm{M}$ phosphate buffer at $\mathrm{pH} 7$ and adjusted to an osmolarity of approximately 800 mosm with the addition of $\mathrm{NaCl}$. Primary fixation was performed on ice and stopped after $45 \mathrm{~min}$ with three buffer rinses for 10, 15, and $20 \mathrm{~min}$. For post-fixation, $1 \% \mathrm{OsO}_{4}$ in $0.1 \mathrm{M}$ phosphate buffer was used for $30 \mathrm{~min}$ on ice. The pericardial bodies were washed in double-distilled water, and were dehydrated in a graded ethanol series followed by propylene oxide and subsequent embedding in Araldite resin. The sections were cut using a diamond knife. Ultrathin sections $(60 \mathrm{~nm})$ were stained with uranyl acetate and lead citrate, and were analyzed using a Zeiss 900 transmission electron microscope. Images were recorded with a Wideangle Dual Speed 2 k-CCD-Camera.

\section{Light Microscopy}

Small individuals of Ciona intestinalis were fixed in Bouin's fixative, an aqueous solution containing $8 \%$ formaldehyde, $5 \%$ acetic acid and $1 \%$ picric acid, for $48 \mathrm{~h}$, dehydrated through an ethanol series, followed by paraffin-embedding in an automatic tissue processor (Shandon Hypercenter XP). Samples were subsequently sectioned at $10 \mu \mathrm{m}$ thickness using a rotary microtome. The sections were mounted on glass slides and stained according to the Masson-Goldner-trichrome method.

Semi-thin sections $(1 \mu \mathrm{m})$ of araldite-embedded pericardial bodies (see section "Electron Microscopy") were stained with toluidine blue.

Different tissue samples (branchial basket, intestinal tract, and body wall) of $C$. intestinalis were smeared directly on glass slides in a drop of seawater and examined for signs of parasite infections. These samples were observed either without fixation and without staining or after Giemsa staining. 
Light microscopical examination was carried out on a Zeiss-Axioplan microscope equipped with a Canon Eos 700D.

\section{Sequence Analysis}

\section{Isolation of DNA, amplification by PCR, and sequencing}

Genomic DNA was extracted from several tunicate tissues, specifically branchial basket, intestinal tract, body wall, and pericardial body. Tissues were cut into small pieces with a scalpel, followed by grinding with a pestle. Next, DNA was isolated with a QIAgen DNAeasy Kit, applying the animal tissue protocol after ATL-proteinase K digestion overnight. DNA content and quality were measured with a photospectrometer (NanoDrop). Polymerase chain reactions (PCR) were performed using a QIAGEN TopTaq Master Mix with 2-3 $\mu$ l of genomic DNA as a template, and $1 \mu$ of each primer $(10 \mathrm{mM})$. In a nested PCR approach, to detect infections with marine gregarines, we first amplified SSU 18SrRNA with the generic primers F1 (GCGCTACCTGGT TGATCCTGCC) and R1 (GATCCTTCTGCAGGTTCA CCTAC) for eukaryotes [18] and then amplified $1 \mu$ of the first product as a template with primers F2 (GTDAATCGG CGTGTTCYACG) and R2 (GAATGCCCTCARCCGTTC) specific for lecudinid marine gregarines [29]. After visual analysis on agarose gels, PCR products of the expected size (128 bp) were sequenced at LGC genomics using both amplification primers. The identity of the gregarine sequences was verified to genus level via comparisons to reference sequences by NCBI BLAST.

\section{Results}

In the pericardium of the ascidian model organism Ciona intestinalis, a white globular structure, the pericardial body, is regularly found (Fig. 1). Because two hypotheses regarding the function of the pericardial body exist, we combined morphological and molecular approaches in order to correlate parasite infection with an assessment of functional structure of the pericardial body.

\section{Gross examination}

Eleven specimens of Ciona intestinalis were anaesthetized and their pericard-heart complexes dissected. A white pericardial body of irregular, often roughly oval shape and measuring approximately $100-250 \mu \mathrm{m}$, was present in each individual (Fig. 1c). For each individual, we dissected the pericardial bodies and either processed them for transmission electron microscopy $(n=4)$ or light microscopy $(n=5)$. In addition, we macerated tissue combined from the branchial basket, intestinal tract, and body wall of each individual $(n=11)$ for light-microscopic inspection and molecular analyses. Two pericardial bodies were also used for molecular analyses.

\section{Parasites}

The light microscopic inspection of the macerated tissues (branchial basket, intestinal tract, and body wall) showed that each individual was infected with parasitic gregarines (Fig. 2). The gregarines measured approximately $80 \mu \mathrm{m}$ in length, were of pear shape to elongated shape, and featured a round prominent nucleus of approximately $8 \mu \mathrm{m}$ diameter. Giemsa staining enhances visibility, but shrinks the gregarines to approximately $80 \%$ in linear dimensions. The distinction between a broader, blunter posterior end, and a narrower anterior tip characterized the gregarines as trophozoites.

Genomic DNA was isolated from the dissected tissues (branchial basket, intestinal tract, body wall, and pericardial body) and subjected to PCR amplification. Gregarinespecific primers [29], amplified s a $128 \mathrm{bp}$ fragment of the 18SrDNA sequence from DNA isolated from branchial basket, intestinal tract, and body wall tissue, but not from pericardial body tissue. Of these fragments, 62-63 bp were high quality sequences in 5 of the 11 ascidians that were used in BLAST alignments (Figure S1, Supplementary Data). BLAST searches of these five sequences against the nucleotide database at NCBI resulted in 98\% (2e-21-6e-21) sequence identity with only 1 consistent base mismatch and Lankesteria ascidae 18SrRNA (Gene Bank Sequence ID: JX187607 from position 765 to 826) as the only hit. BLAST searches with the sequences of the remaining 6 specimens yielded hits with two different species within the gregarine genus Lankesteria: L. ascidiae $(n=2,2 \mathrm{e}-15-2 \mathrm{e}-17,96 \%$ identity) and $L$. cystodytes $(n=4,2 \mathrm{e}-14-2 \mathrm{e}-15,96 \%$ identity). Both gregarine species have been isolated from ascidians from the Pacific Ocean, $L$. ascidiae from $C$. intestinalis $[21,24]$.

\section{Pericardial bodies}

\section{Histology}

Light microscopic observation of paraffin-embedded pericardial bodies, stained with Masson-Goldner-trichromatic stains and sectioned at $10-\mu \mathrm{m}$ thickness, showed that the pericardial bodies consisted of a conglomerate of cells and cellular fragments of various size and shapes (Fig. 1). Roundish to globular cells of approximately $10-\mu \mathrm{m}$ diameter with dark brownish nuclei of around 5- $\mu \mathrm{m}$ diameter are numerous. The cytoplasm of these globular cells is predominantly greenish in coloration, indicating proteinaceous content at 


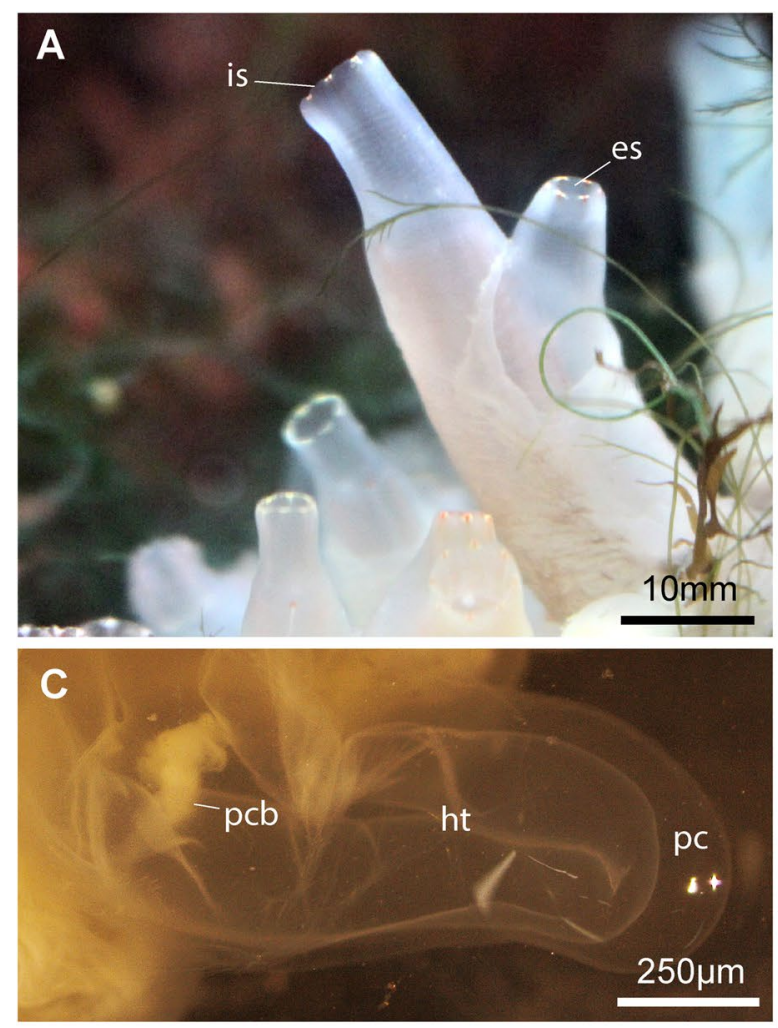

B

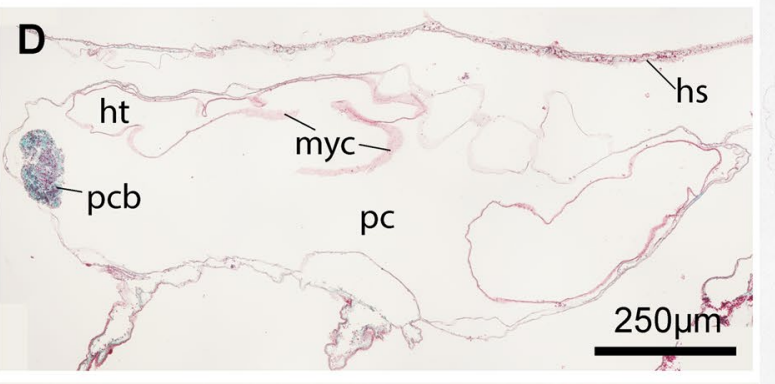

E
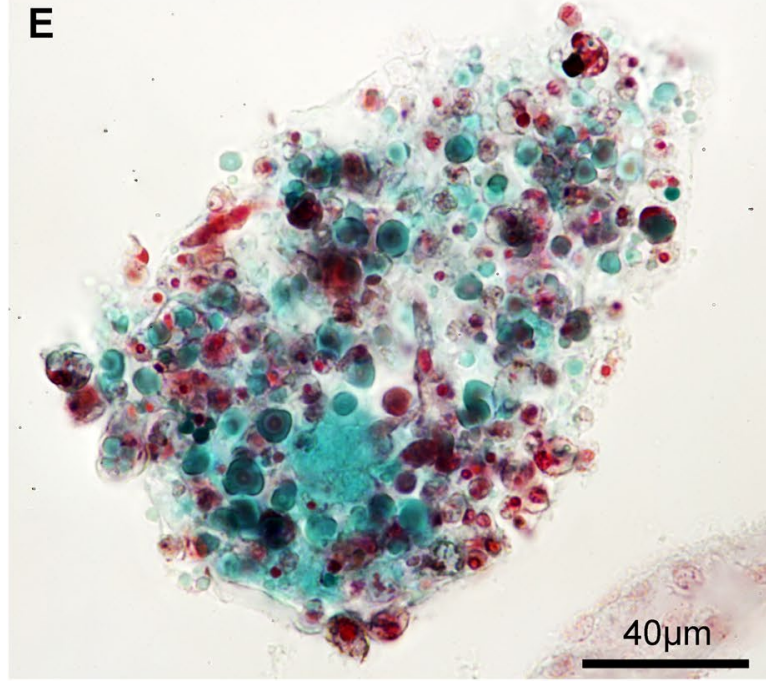

$\mathrm{bb}$ 
4Fig. 1 Pericardial body within the heart-pericard complex in the ascidian Ciona intestinalis. a Healthy, live individuals in feeding position show relaxed and opened siphons. Note colored eyespots around incurrent and excurrent siphons. b Light-micrograph of a parasagittal section showing inner anatomy. The pericardial body (pcb) is seen as a whitish structure of irregular shape. c Freshly dissected heart-pericard complex (h/pc). d Histological section of a h/pc sectioned along the longitudinal axis. e Light micrographic detail of histological section of a pcb. Note numerous round cells of different sizes, elongated cellular elements, and cellular fragments. at atrium, $b b$ branchial basket, es excurrent siphon, eso esophagus, $h / p c$ heartpericard-complex, $h s$ horizontal septum, $h t$ heart, is incurrent siphon, $p c b$ pericardial body, $s t$ stomach, $t u$ tunic

a lower $\mathrm{pH}$. Elongated cells of $20 \mu \mathrm{m}$ length and a diameter of 5-8 $\mu \mathrm{m}$ are also regularly encountered. The cytoplasm of these elongated cells is reddish in coloration, and the nucleus is darker stained. There are also large aggregations of cellular material, sometimes with several nuclei. Besides the larger globular cells, globular cells of merely $5 \mu \mathrm{m}$ in diameter are numerous. The coloration of the globular cells is varied; the cytoplasm is mostly greenish, but sometimes transparent and numerous vesicles of different colors are present.

In semithin sections $(0.7 \mu \mathrm{m})$ stained with toluidine blue, the elongated cells often feature a repeated banding pattern of dark and light stripes (Fig. 3a), typical of sarcomeres of myocytes. In these histological preparations, several of such myocytes, with more or less distinct bands of sarcomeres are regularly found clumped together. In some of these myocytes, nuclei show the normal pattern of homogenous light blue euchromatin and darker spots of heterochromatin, whereas some nuclei appear unusually light with granular specks. In the latter cells, the cytoplasm shows vacuolated areas and degenerating organelles. Two kinds of other cells can be discerned: A conspicuous cell type with a single larger vacuole. This vacuole limits the cytoplasm to a thin margin between vacuole and cell membrane giving the cell a ring like appearance in cross section. With the nucleus protruding from this thin area, the appearance is more accurately described as that of a signet ring (Figs. 3a, 4c). The other cell type usually possesses several smaller vacuoles that appear empty and numerous dark granules that are evenly stained (Fig. 4a).

\section{Ultrastructure}

\section{Muscle Cells}

Transmission electron microscopy revealed that the myocytes were at different stages of degeneration. The typical banding pattern of the sarcomeres was still discernible due to the repeated occurrence of darker $z$ lines. The length of a sarcomere, however, differed between cells, ranging from $0.2 \mu \mathrm{m}$ to more than $1 \mu \mathrm{m}$, with the $z$ lines in various stages of disintegration, becoming successively more dilated (Fig. 3b). In some of the degenerating myocytes, myosin fibers could be identified, although the arrangement in sarcomeres was no longer recognizable (Fig. 2c). Dilatation and confluence of elements lead to internal fragmentation. Lysosomes, areas with vesicularized membrane-bound irregular bodies, and degenerating organelles, which are mostly mitochondria, if discernible, predominate the cytoplasm of these degenerating myocytes.

\section{Hemocytes}

Between the degenerating myocytes, two other cell types are seen: granular amebocytes and signet ring cells. Granular amebocytes (Fig. 4b) possess several electron lucent vacuoles with little precipitation as content and numerous granules with more electron dense content. In these granular amebocytes the periphery of the cells is extended into pseudopods. Electron microscopy clearly illustrates the morphology of the signet ring cells (Fig. 4d). These signet ring cells show a single larger vacuole, a narrow rim of cytoplasm, and a protruding nuclear region, and adds the occurrence of pseudopods in this cell type as well (Fig. 4d).

No signs of parasites could be discovered in histological preparations and were also not found in the ultrastructural preparations (see Figs. 3, 4).

\section{Discussion}

According to phylogenomic analyses, tunicates comprise the most likely sister group to vertebrates within chordates [10, 15], with typical chordate characters such as a dorsal brain, a larval postanal tail in most species, and a branchial basket. Like all chordates, tunicates possess a heart surrounded by a pericardium on the ventral side, immediately posterior to the branchial basket $[2,14]$. Based on these and additional developmental similarities, the tunicate heart is considered a homolog of the vertebrate heart $[1,8,30]$. Most tunicates are sessile ascidians and some ascidian species possess a pericardial body, a little white ball, found within the pericardium. It is regularly found in Ciona spp., but has also been reported occasionally in other species in the family Ascidiaceae (e.g., Fernandez 1907; Kalk [14]. Regarding the functional significance of this pericardial body two main hypotheses have been suggested: hypothesis $1\left(\mathrm{H}_{1}\right)$ suggests that the pericardial body is a site of degradation of discarded myocardial cells (e.g., Roule [2], Millar [23], Burighel and Cloney [27]), whereas according to hypothesis $\left(\mathrm{H}_{2}\right)$ the pericardial body functions in parasite removal $[31,32]$. 
Fig. 2 Light micrographs of gregarines in branchial basket tissue preparations. a, b living specimens. c, d Giemsa staining enhances visibility of the nucleus (nu)
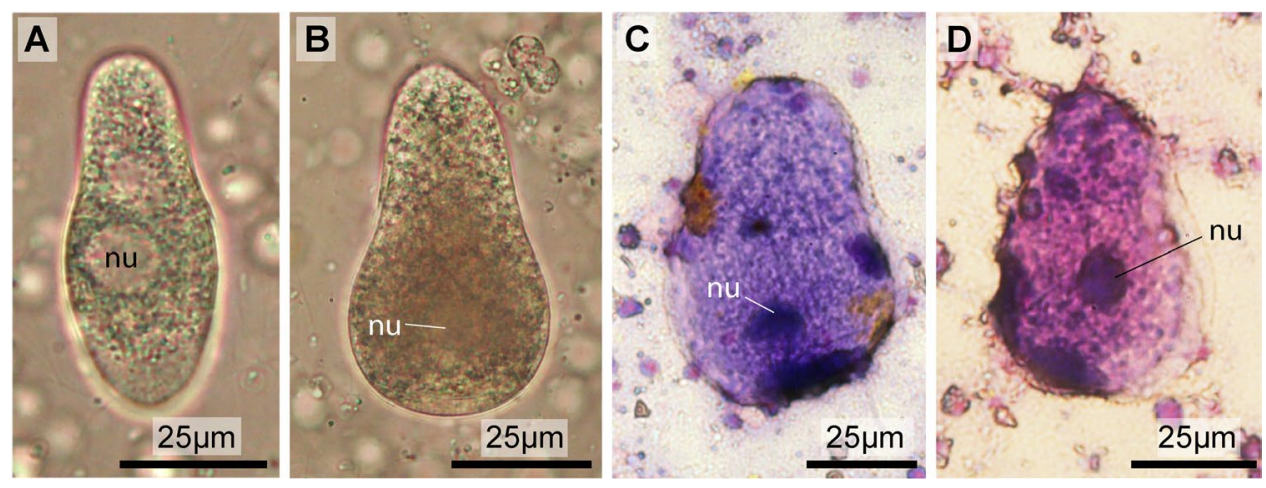
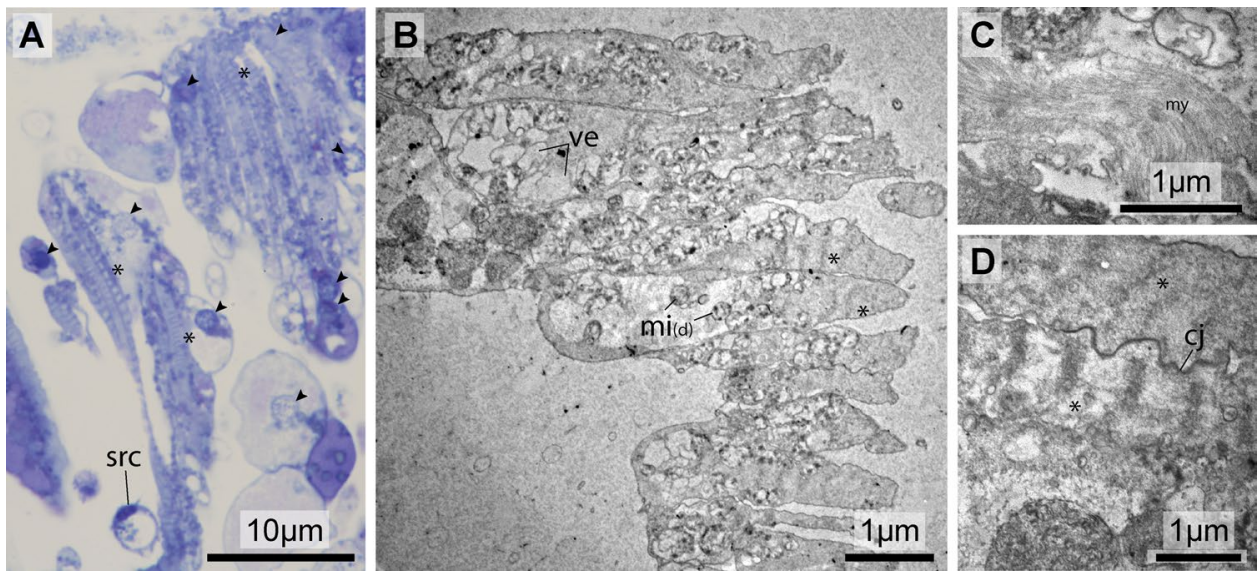

Fig. 3 Histological and ultrastructural details of the pericardial body. a Semithin section stained with toluidine blue showing hemocytes and myocardial cells. b Transmission electron micrograph of partly degenerated myocardial cells. c Myofilaments in degenerating myo- cardial cell. d Detail of sarcomere in a degenerating myocardial cell. $c j$ cell junction, $m i_{(d)}$ degenerating mitochondria, $m y$ ofilaments, $s r c$ signet ring cell, ve -vesicles; arrowheads: nuclei, asterisks: areas with sarcomeres
In principal, these hypotheses should be discernible by simultaneously investigating the infection status of specimens and the ultrastructure of the pericardial body in infested individuals: we argue that in infected specimens, there should be ultrastructural evidence of degenerating muscle cells in the pericardial body, if $\mathrm{H}_{1}$ is true. If, on the other hand, $\mathrm{H}_{2}$ is correct, then in infected specimens, there should be ultrastructural evidence of parasites in the pericardial body.

We investigated a total of 11 specimens and found that every individual was infested with apicomplexan parasites, a prevalence of $100 \%$. Apicomplexan parasites have been reported from different anatomical compartments in their respective hosts, e.g., from the intestinal tract [5, 26], coelomic cavities, or the hemocoel $[11,28]$. Based on the sequence comparison of a partial region of the 18S rDNAgene, we could identify the parasites as species within the intestinal parasite gregarine genus Lankesteria, most probably L. ascidiae nested within Apicomplexa (e.g., Leander [17], Morrison [25]). Despite the relative shortness of the partial 18S rRNA-gene we amplified, we are confident that the gregarines belong to the genus Lankesteria, while the species attribution remains more capricious. We analyzed the ultrastructure of the pericardial bodies of individuals, all of which were infected with Lankesteria spp., and did not find evidence of parasites within the pericardial bodies. Therefore, we conclude that $\mathrm{H}_{2}$ is not supported by this study, and we strengthen $\mathrm{H}_{1}$, the hypothesis that the pericardial body is a site of removal of accumulating, degenerating apoptotic myocardial cells within the pericardium. Of course, this does not preclude the possibility that other gregarine parasites infiltrate other body compartments, such as the hemocoel [11], coelomic cavities in general [19], or specifically the pericardium as was observed by Scippa et al. [32] and Cianco et al. [6]. This would of course be the case for the intracellular parasites, such as Cardiosporidium cionae, observed by these authors within hemocytes in the pericardium $[6,32]$. We could not find histological or ultrastructural evidence of such an intracellular infestation. Moreover, our attempts to identify an infection with $C$. cionae by 
Fig. 4 Histological and ultrastructural details of hemocytes within the pericardial body. a, c Semithin section stained with toluidine blue. $\mathbf{b}$, $\mathbf{d}$ Transmission electron micrographs. a, b: Granular amoebocytes. c, d: Signet ring cells. $c f$ cell fragment, $g r$ granulum, $n u$ nucleus, pp pseudopodium, $v a$ vacuole
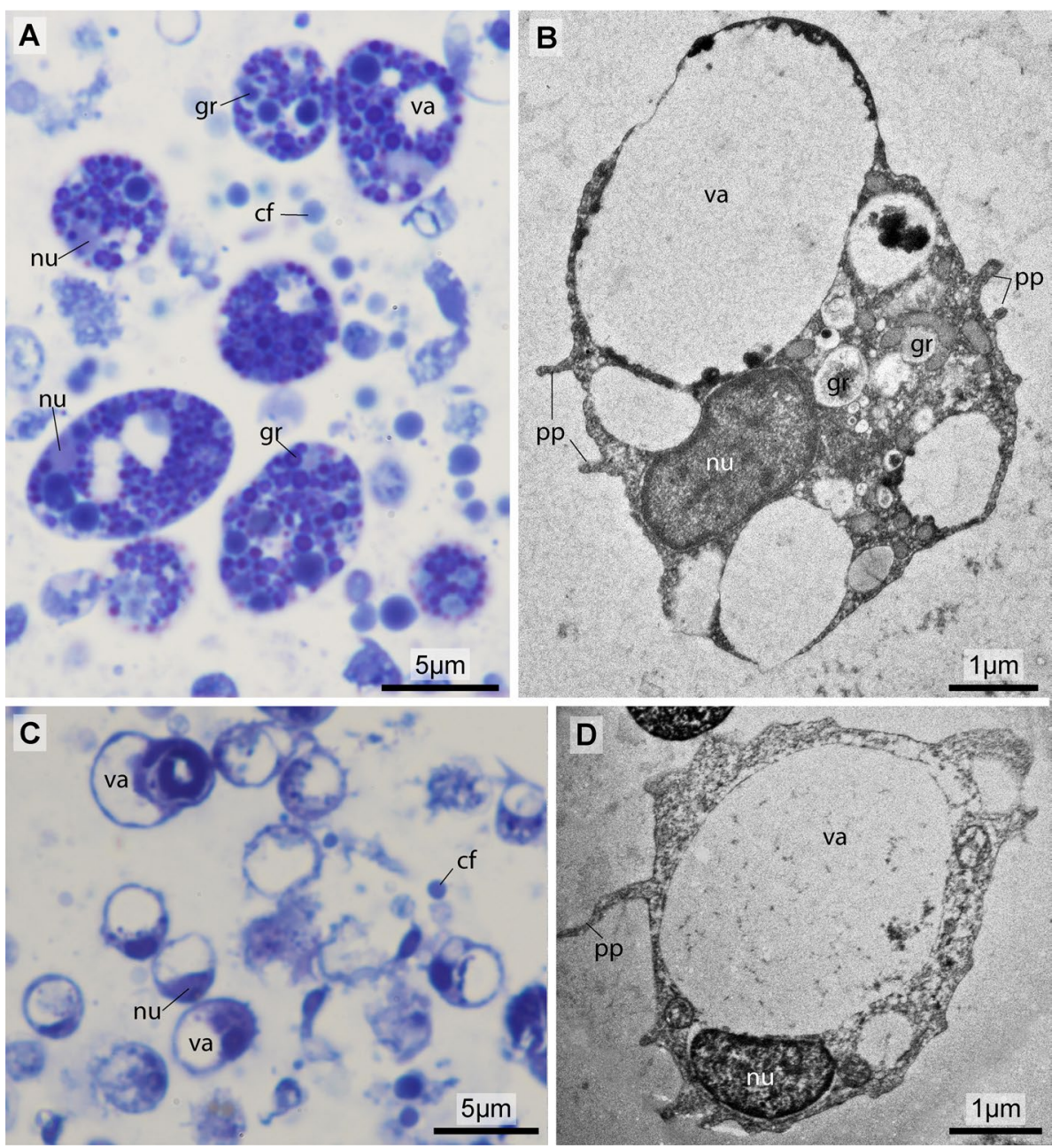

amplifying a partial sequence of the 18srRNA gene using a nested approach by first amplifying SSU 18SrRNA with the generic primers F1 (GCGCTACCTGGTTGATCCTGCC) and R1 (GATCCTTCTGCAGGTTCACCTAC) for eukaryotes [18] and then using the product as a template with primers designed to specifically amplify $C$. cionae 18 SrRNA (GenBank accession: EU052685) could not pinpoint such an infestation.

Similar to the findings reported by Scippa and Izzo [31], we observed hemocytes in the pericardium. We identified granular amebocytes and signet-ring cells in the present study, and these hemocytes possess pseudopodial extensions, are capable of phagocytosis (e.g., de Leo [2, Burighel and Cloney [9]), and engulf the apoptotic cardiomyocytes and their remnants. Hemocyte-mediated phagocytosis is the primary innate immune defense of ascidians [7], and these can serve roles in parasite removal $[4,9]$.

For the electron and light microscopic investigation, we intentionally selected smaller, and thus younger individuals. In these smaller and younger specimens, parasites might not have penetrated into the pericardial coelomic cavity in high enough numbers to be detected in light microscopic, histological, or electron microscopic preparations. This line of reasoning, could reconcile the two hypotheses $\mathrm{H}_{1}$ and $\mathrm{H}_{2}$ : the primary function of the pericardial body is seen in the removal of discarded, apoptotic myocardial cells $\left(\mathrm{H}_{1}\right)$, which is facilitated by hemocytes. These hemocytes can also serve as a line of defense, in case parasites infect the coelomic cavity of the pericard as has been shown by Scippa et al. [32]. In conclusion, we strengthen the case in favor of hypothesis $\mathrm{H}_{1}$, i.e., that the main and primary function of the pericardial body lies in the removal of degenerating myocytes, but the phagocytic hemocytes involved in this process might also take up parasites in the pericardium. In any case, despite its didactic value, we suggest that in the next edition of the textbook "Zoologisches Praktikum" the words "unbekannter Funktion" (= of unknown function) are omitted when referring to the pericardial body.

Incidentally, the observation of hemocytes in the pericardium indicates that the cells are capable of leaving the blood stream and migrating through epithelially organized pericardial cells to reach the sites where they fulfill their functional 
roles. This cellular behavior is similar to leucocytes in vertebrates, where this behavior is termed diapedesis (e.g., Marchesi and Florey [13], Filippi [22]. The confirmation of apoptotic cardiomyocytes in the pericardial body also implies a general turnover of these muscle cells in the heart of $C$. intestinalis. While molecular details of such a process are known in vertebrates (e.g., Takemura et al. [36], Vujic et al. [37]) and invertebrate model organisms (e.g., Zhu et al. [38]), hardly anything seems to be known of this process in marine invertebrates. The report of differentially proliferating and apoptotic heart muscle cells in two bivalve species [35], however, indicates that the tightly regulated turnover of heart muscle cells is a widespread phenomenon. The heart of $C$. intestinalis; therefore, is a promising model to elucidate this process also from an evolutionary and molecular perspective.

Acknowledgements We thank Kai Matuschewski for supporting all aspects of our research and for providing an excellent research environment. We are especially grateful to Peer Martin for excellent technical assistance, to Juliane Schaer for valuable suggestions concerning molecular analyses, and to Alyssa Ingmundson for greatly improving the manuscript.

Funding Open Access funding enabled and organized by Projekt DEAL.

\section{Compliance with ethical standards}

All authors have read, understood, and consented to the submitted version of the manuscript.

Conflict of interest No conflicts of interest exist.

Human and animal rights All animal welfare regulations were followed.

Ethical approval The research performed for manuscript ACTPD-20-00293 does not constitute animal experimentation according to German laws.

Open Access This article is licensed under a Creative Commons Attribution 4.0 International License, which permits use, sharing, adaptation, distribution and reproduction in any medium or format, as long as you give appropriate credit to the original author(s) and the source, provide a link to the Creative Commons licence, and indicate if changes were made. The images or other third party material in this article are included in the article's Creative Commons licence, unless indicated otherwise in a credit line to the material. If material is not included in the article's Creative Commons licence and your intended use is not permitted by statutory regulation or exceeds the permitted use, you will need to obtain permission directly from the copyright holder. To view a copy of this licence, visit http://creativecommons.org/licenses/by/4.0/.

\section{References}

1. Anderson HE, Christiaen L (2016) Ciona as a simple chordate model for heart development and regeneration. J Cardiovasc Dev Dis 3:25. https://doi.org/10.3390/jcdd3030025

2. Burighel P, Cloney RA (1997) Urochordata: Ascidiacea. In: Harrison FW, Ruppert EE (eds) Microscopic anatomy of Invertebrates Hemichordata, Chaetognatha, and the invertebrate chordates. Wiley-Liss, Incorporation, New York, pp 221-347

3. Brunetti R, Gissi C, Pennati R, Caicci F, Gasparini F, Manni L (2015) Morphological evidence that the molecularly determined Ciona intestinalis type A and type B are different species: Ciona robusta and Ciona intestinalis. J Zool Syst Evol Res 53:186-193. https://doi.org/10.1111/jzs.12101

4. Choi DL, Jee BY, Choi HJ, Hwang JY, Kim JW, Berthe FCJ (2006) First report on histology and ultrastructure of an intrahemocytic paramyxean parasite (IPP) from tunicate Halocynthia roretzi in Korea. DAO 72:65-69

5. Ciancio A, Scippa S, Cammarano M (2001) Ultrastructure of trophozoites of the gregarine Lankesteria ascidiae (Apicomplexa: Eugregarinida) parasitic in the ascidian Ciona intestinalis (Protochordata). Eur J Protistol 37:327-336

6. Ciancio A, Scippa S, Finetti-Sialer M, De Candia A, Avallone M, De Vincentiis M (2008) Redescription of Cardiosporidium cionae (Van Gaver and Stephan, 1907) (Apicomplexa: Piroplasmida), a plasmodial parasite of ascidian haemocytes. Europ J Protistol 44:181-196. https://doi.org/10.1016/j.ejop.2007.11.005

7. Cima F, Franchi N, Ballarin L (2016) Chapter 2-origin and functions of tunicate hemocytes. In: Malagoli D (ed) The evolution of the immune system. Conservation and Diversification. Academic Press, London, pp 29-49

8. Davidson B, Levine M (2003) Evolutionary origins of the vertebrate heart: Specification of the cardiac lineage in Ciona intestinalis. PNAS 100:11469-11473. https://doi.org/10.1073/ pnas. 1634991100

9. de Leo G (1992) Ascidian hemocytes and their involvement in defence reactions. Boll zool 59:195-214. https://doi. org/10.1080/11250009209386669

10. Delsuc F, Philippe H, Tsagkogeorga G, Simion P, Tilak M-K, Turon X, López-Legentil S, Piette J, Lemaire P, Douzery EJP (2018) A phylogenomic framework and timescale for comparative studies of tunicates. BMC Biol 16:39. https://doi.org/10.1186/ s12915-018-0499-2

11. Desportes I, Schrével J (2013) Marine gregarines. In: Desportes I, Schrével J (eds) Treatise on zoology - anatomy, taxonomy, biology. The gregarines, vol 1. Brill, Leiden, Boston, pp 197-375. https://doi.org/10.1163/9789004256057_005

12. Fernandez M (1906) Zur Kenntnis des Pericardkörpers einiger Ascidien. Jenaische Zeitschrift für Naturwissenschaft 4(1-1):8

13. Filippi M-D (2016) Chapter two-mechanism of diapedesis: importance of the transcellular route. In: Alt FW (ed) Advances in immunology, vol 129. Academic Press, London, pp 25-53. https ://doi.org/10.1016/bs.ai.2015.09.001

14. Kalk M (1970) The organization of a tunicate heart. Tissue 99:1-18

15. Kocot KM, Tassia MG, Halanych KM, Swalla BJ (2018) Phylogenomics offers resolution of major tunicate relationships. Mol Phyl Evol 121:166-173. https://doi.org/10.1016/j.ympev .2018.01.005 
16. Kükenthal WG (1898) Leitfaden für das zoologische Praktikum. Gustav Fischer, Jena

17. Leander BS (2008) Marine gregarines: evolutionary prelude to the apicomplexan radiation? Trends Parasitol 24:60-67. https:// doi.org/10.1016/j.pt.2007.11.005

18. Leander BS, Clopton RE, Keeling PJ (2003) Phylogeny of gregarines (Apicomplexa) as inferred from SSU rDNA and beta-tubulin. Int J Syst Evol Bicrobiol 53:345-354. https://doi.org/10.1099/ ijs.0.02284-0

19. Leander BS, Shane AJL, Wyth M, Landers SC (2006) Phylogeny of Marine Gregarines (Apicomplexa)—Pterospora, Lithocystis and Lankesteria - and the Origin(s) of Coelomic Parasitism. Protist 157:45-60. https://doi.org/10.1016/j.protis.2005.10.002

20. Lemaire $P$ (2011) Evolutionary crossroads in developmental biology: the tunicates. Development 138:2143-2152

21. Levine ND (1981) New Species of Lankesteria (Apicomplexa, Eugregarinida) from Ascidians on the Central California Coast. Profozool 28:363-370. https://doi.org/10.1111/j.1550-7408.1981. tb02868.x

22. Marchesi VT, Florey HW (1960) Electron micrographic observations on the emigration of leucocytes. Quart J Exp Physiol Cog Med Sci 45:343-375. https://doi.org/10.1113/expphysiol.1960. sp001489

23. Millar RH (1953) Ciona.-L.M.B.C. Mem Typical Br Mar Plants aAnim 35:1-123

24. Mita K, Kawai N, Rueckert S, Sasakura Y (2012) Large-scale infection of the ascidian Ciona intestinalis by the gregarine Lankesteria ascidiae in an inland culture system. DAO 101:185-195. https://doi.org/10.3354/dao02534

25. Morrison DA (2009) Evolution of the Apicomplexa: where are we now? Trends Parasitol 25:375-382. https://doi.org/10.1016/j. pt.2009.05.010

26. Ormières R (1965) Recherches sur les sporozoaires parasites des Tuniciers. Vie et Milieu 15:823-946

27. Roule L (1884) Recherches sur les Ascidies Simples des cotes de Provence. Phallusiadées. Monographie de la Ciona intestinalis. Ann Mus Hist Nat Marseille Zool 1:1-270

28. Rueckert S, Leander BS (2009) Morphology and phylogenetic position of two novel marine gregarines (Apicomplexa,
Eugregarinorida) from the intestines of North-eastern Pacific ascidians. Zool Scripta 37:637-645. https://doi.org/10.111 1/j.1463-6409.2008.00346.x

29. Rückert S, Wakeman Jenke-Kodama KCH, Leander BS (2015) Molecular systematics of marine gregarine apicomplexans from Pacific tunicates, with descriptions of five novel species of Lankesteria. Int J Syst Evol Microbiol 65:2598-2614. https://doi. org/10.1099/ijs.0.000300

30. Satou Y, Satoh N (2006) Gene regulatory networks for the development and evolution of the chordate heart. Genes Dev 20:2634 2638. https://doi.org/10.1101/gad.1485706

31. Scippa S, Izzo C (1996) An ultrastructural study of the hemocytes of the pericardial body in the ascidian Ciona intestinalis (L.). Acta Zool 77:283-286

32. Scippa S, Ciancio A, deVincentiis M (2000) Observations on an apicomplexan microparasite from the pericardic body of Ciona intestinalis L. (Protochordata). Europ J Protistol 36:85-88

33. Stach T (2016) Potato, potahto, Tomato, tomahto: Let's call the whole thing off. Evol Dev 18:69-70. https://doi.org/10.1111/ ede. 12178

34. Storch V, Welsch U (2014) Kükenthal Zoologisches Praktikum. Springer Spektrum, Berlin

35. Strahl J, Abele D (2010) Cell turnover in tissues of the longlived ocean quahog Arctica islandica and the short-lived scallop Aequipecten opercularis. Mar Biol 157:1283-1292. https://doi. org/10.1007/s00227-010-1408-6

36. Takemura G, Kanoh M, Minatoguchi S, Fujiwara H (2013) Cardiomyocyte apoptosis in the failing heart-a critical review from definition and classification of cell death. Int J Cardiol 167:23732386. https://doi.org/10.1016/j.ijcard.2013.01.163

37. Vujic A, Natarajan N, Lee RT (2020) Molecular mechanisms of heart regeneration. Semin Cell Dev Biol 100:20-28. https://doi. org/10.1016/j.semcdb.2019.09.003

38. Zhu S, Han Z, Luo Y, Chen Y, Zeng Q, Wu X, Yuan W (2017) Molecular mechanisms of heart failure: insights from Drosophila. Heart Fail Rev 22:91-98. https://doi.org/10.1007/s1074 1-016-9590-3

Publisher's Note Springer Nature remains neutral with regard to jurisdictional claims in published maps and institutional affiliations. 